\title{
Correction to: Association between intraoperative hyperglycemia and postoperative end-organ dysfunctions after cardiac surgery: a retrospective observational study
}

\author{
Shinsaku Matsumoto ${ }^{1,2}\left(\right.$ Diroki Omiya ${ }^{1} \cdot$ Waso Fujinaka ${ }^{1} \cdot$ Hiroshi Morimatsu $^{2}$
}

Published online: 4 December 2021

(c) The Author(s) under exclusive licence to Japanese Society of Anesthesiologists 2021

\section{Correction to: Journal of Anesthesia \\ https://doi.org/10.1007/s00540-021-03024-5}

In the original publication of the article, second affiliation was not tagged to corresponding author and affiliations are correctly tagged in this correction.

The original article was updated.

Publisher's Note Springer Nature remains neutral with regard to jurisdictional claims in published maps and institutional affiliations.

The original article can be found online at https://doi.org/10.1007/ s00540-021-03024-5.

Shinsaku Matsumoto shinsaku823@yahoo.co.jp

1 Department of Anesthesiology and Intensive Care, Hiroshima City Hiroshima Citizens Hospital, Hiroshima, Japan

2 Department of Anesthesiology and Resuscitology, Okayama University Graduate School of Medicine, Dentistry, and Pharmaceutical Sciences, Okayama, Japan 